\title{
The Variation Law of Mechanical Parameters of Type I Fatigue Crack Tip Under High Frequency Resonant Loading
}

\author{
Gao Hong-Li, Zheng Huan-Bin*, Qiu Xin-Guo, Liu Huan and Liu Hui \\ College of Mechanical Engineering, Zhejiang University of Technology, Hangzhou, Zhejiang, 310014, China
}

\begin{abstract}
This work explores the variation law of mechanical parameters at fatigue crack tip of compact tension specimen with type I pre-notch based on dynamic finite element method (FEM) in the high frequency resonant fatigue crack propagation test. The displacement fields, the strain fields and the stress intensity factors (SIF) at CT specimen fatigue crack tip in one stress cycle and at different crack lengths under constant amplitude high frequency sinusoidal alternating loading condition are calculated and the related variation laws of mechanical parameters are analyzed. In order to calculate the dynamic SIF at fatigue crack tip, the static SIF has been calculated first. The compared results of the static finite element analysis with the theoretical calculation show that finite element modeling and calculating method and respective results are accurate. Secondly, the variation law of SIF at crack tip during the process of fatigue crack propagation test is studied by dynamic FEM. Finally, the high frequency resonant fatigue crack propagation test has been performed and the dynamic strain gauge is used to measure the strain at crack tip during one stress cycle. The research results show that during crack stable propagation stage, the displacement, strain and SIF at type I fatigue crack tip are in the same form having high frequency resonant load, and the displacement, strain and SIF amplitude increase with the crack growth. The error of static SIF between the calculated result by FEM and the theoretical result is $2.51 \%$. The maximum error of the strain at crack tip between the FEM calculating result and the experimental result is $2.93 \%$.
\end{abstract}

Keywords: Displacement, Dynamic FEM, Fatigue crack tip, High frequency resonant load, Strain field, Stress intensity factor.

\section{INTRODUCTION}

In general, fatigue failure is the main failure mode of the mechanical parts. Fatigue crack initiation and propagation in the material or structure occur when they are subjected to alternating loading, and also can result in material fatigue failure [1-3]. Nowadays, the fatigue failure cannot be completely studied by the effective theoretical method, so, the specific materials for fatigue crack propagation test have acquired considerable importance for studying the fracture mechanism. They have great significance in the improvement of the reliability and the service life of mechanical products $[4,5]$. Most common crack formed in the practical engineering is type I crack which is also the most dangerous crack type. The fatigue crack propagation tests of compact tension specimens with type I pre-notch are performed to study the fatigue crack growth behavior of material by measuring the relationship between the fatigue crack growth rate and SIF amplitude under the alternating loading condition. It is shown that SIF is an important parameter for calculating the fatigue crack growth rate and judging whether structure has crack failure [6]. However, the initiation, growth and fracture of the fatigue crack cannot be completely characterized by the shape and size of macroscopic crack. In order to study the mechanism of fatigue crack propagation comprehensively and systematically, not only the study on the relationship between the fatigue crack growth rate and

*Address correspondence to this author at the College of Mechanical, Engineering, Zhejiang University of Technology, Zhejiang, 310014, P.R. China; Tel: 150687577571; E-mail: huanbinstrong@126.com
SIF range of macroscopic crack has a significance, but also the study on the mechanical characteristic parameters such as the displacement, strain and the stress field at crack tip during the process of fatigue crack growth is necessary.

The fatigue crack propagation tests including low frequency fatigue test based on electro-hydraulic forced vibrating system whose commonly used vibration frequency is from 1 to $10 \mathrm{~Hz}$, and high frequency fatigue test based on electromagnetic resonance vibrating system whose commonly used vibration frequency is from 50 to $300 \mathrm{~Hz}$, are the equipment to test the fatigue properties of metal materials and components based on resonance principle [7]. This equipment is widely used for fatigue test of materials by mechanics Lab on account of its high testing frequency, low energy consumption, short testing time and good testing waveforms and so on. The statics method is often used to calculate the displacement, strain, stress field and stress intensity factors at crack tip in the electro-hydraulic forced vibrating fatigue crack propagation test. But because of the inertial effect and stress wave spread effect [8-10], the same calculation for the CT specimen which vibrates at high-speed under high frequency resonant loading in the resonant fatigue crack propagation test is more complex. Unfortunately, the analytical solution of dynamic SIF is still not obtained yet. In recent years, many theoretical and experimental studies have concentrated on the displacement, strain field and SIFs at crack tip [11-15], but most of the previous researches were performed under the static and quasi-static situation. In this paper, the dynamic finite element method is used to explore the variation law of the displacement field, the strain field and SIFs at crack tip under constant amplitude high 
frequency sinusoidal alternating loading during the process of fatigue crack propagation, providing the theoretical base for further investigation of the fatigue crack propagation mechanism and the measurement of crack propagation parameters.

\section{HIGH FREQUENCY RESONANT FATIGUE CRACK PROPAGATION TEST}

The electromagnetic resonant fatigue tester is used to test the fatigue properties of metal materials and components based on the resonance principle under high frequency resonant loading. Fig. (1) shows the structure of commonly used resonant fatigue tester PLG-100, which is a three freedom resonant vibrating system. When the excitation frequency of electromagnetic exciter matches the natural frequency of the vibrating system, the resonance occurs and the workbench vibration which applies to the CT specimen is multiplied under the resonance state. The test is performed under the circumstance of low power consumption. Fig. (2) shows the dimensioned drawing of standard CT specimen. Fig. (3) shows the installation drawing of CT specimen. Fig. (4) shows sinusoidal alternating load in the resonant fatigue crack propagation test, where $F_{\max }$ is the maximum load, $F_{\min }$ is the minimum load, $F_{m}$ is the average load, and $F_{a}$ is the amplitude. In a stress cycle, the CT specimen is always in the tensile stress condition in the resonant fatigue crack propagation test. The installation shown in Fig. (3) indicates that the upper semi-cylinder surface of the upper loading hole and the bottom semi-cylinder surface of the under loading hole are the positioning surfaces where the alternating load passes to the CT specimen.

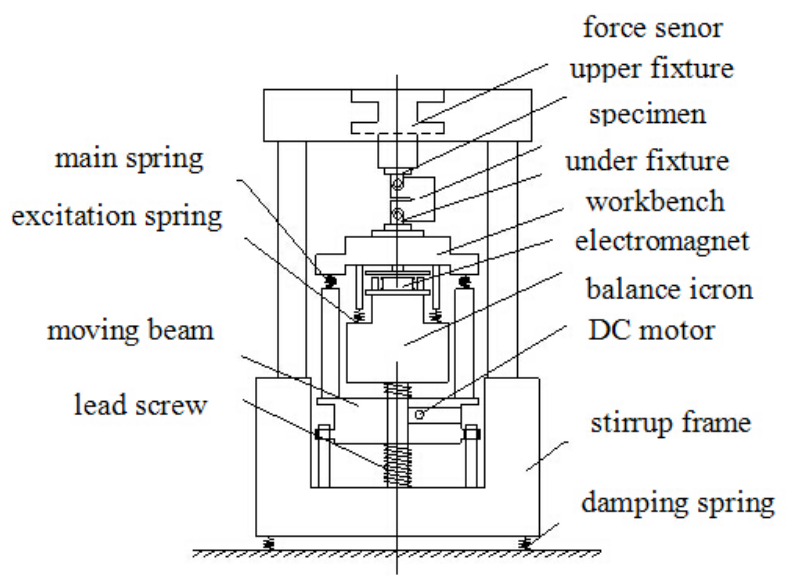

Fig. (1). The structure of resonant fatigue tester.

A series of resonant fatigue crack propagation tests of CT specimen with different materials have been carried out on the testing platform as shown as Fig. (1). The experimental results show that the resonant frequency of the system is from $90 \mathrm{~Hz}$ to $135 \mathrm{~Hz}$ in the stable crack propagation stage. In order to simplify the calculation process, $125 \mathrm{~Hz}$ of sinusoidal alternating load is used for exploring the changing law of the displacement, strain fields and SIFs at crack tip under high frequency resonant loading condition. $125 \mathrm{~Hz}$ is also the nature frequency of the resonant vibrating system with the $5 \mathrm{~mm}$ length fatigue crack standard CT specimen.

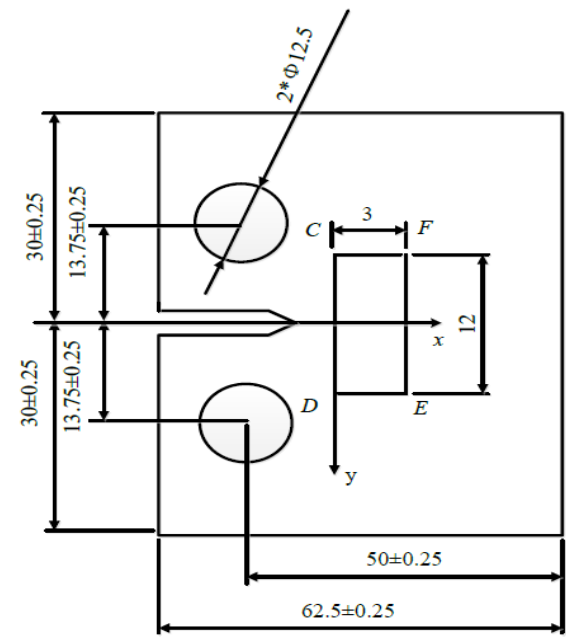

Fig. (2). The dimensioned drawing of CT specimen.

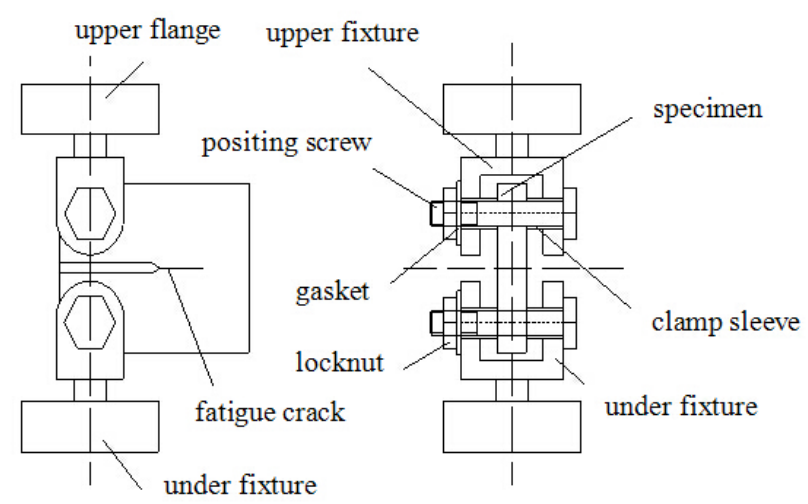

Fig. (3). The installation drawing of CT specimen.

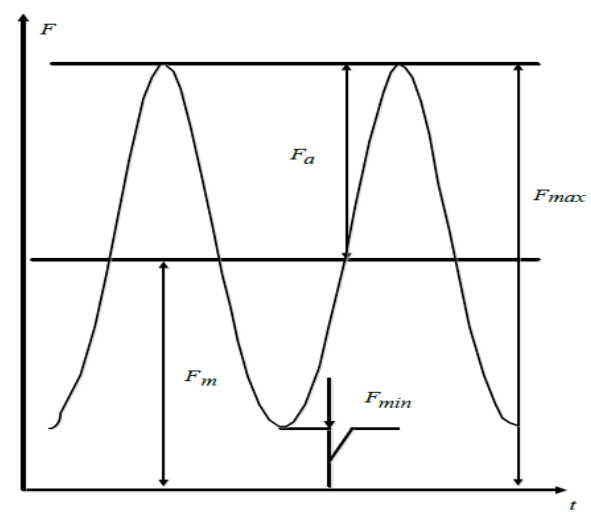

Fig. (4). Sinusoidal alternating load.

\section{THE VARIATION LAW OF SIF AT CRACK TIP UNDER HIGH FREQUENCY RESONANT LOADING CONDITION}

\subsection{The Calculation Method of Dynamic SIF}

The local coordinate system of type I crack tip is shown in Fig. (5). The displacement equations of points around a crack tip are expressed as: 


$$
\begin{aligned}
& u(t)=\frac{K_{I}(t)}{2 G} \sqrt{\frac{r}{2 \pi}} \cos \frac{\theta}{2}\left[(k-1)+2 \sin ^{2} \frac{\theta}{2}\right] \\
& v(t)=\frac{K_{I}(t)}{2 G} \sqrt{\frac{r}{2 \pi}} \sin \frac{\theta}{2}\left[(k+1)-2 \cos ^{2} \frac{\theta}{2}\right]
\end{aligned}
$$

Where $r, \theta$ are the polar coordinates at crack tip; $u(t)$ is the displacement component of $\mathrm{X}$-direction at time $\mathrm{t}$; $v(t)$ is the displacement component of $\mathrm{Y}$-direction at time $\mathrm{t}$; $G$ is the shear modulus of material; $K_{I}(t)$ is the SIF of type I crack at time $\mathrm{t} ; k$ is the coefficient about Poisson's ratio, the specific relationship is expressed as,

$k=3-4 \mu$

The dynamic analysis method of ANSYS is used to get the dynamic response of the opening displacement on the face of the crack. Hence, $K_{I}(t)$ can be calculated due to the evident crack opening displacement which can be used to get an exact approximation. The displacement equation is calculated by using $\theta=0$ at any time t. The displacement equation at $\theta=0$ can be deduced as follows:

$u(t)=\frac{K_{I}(t)}{2 G} \sqrt{\frac{r}{2 \pi}}$

The $K_{I}(t)$ and $r$ can be fitted by least-square principle, and the dynamic SIF value of $r=0$ will be obtained by $K_{I}(t)-r$ curve. Thus, the dynamic SIF at crack tip is determined.

\subsection{FEM Calculation of Dynamic SIF at Crack Tip}

The solution of dynamic SIF is based on the correct model of the CT specimen. In order to prove the rationality and validity of the FEM model, the static ANSYS -FEM is used to calculate the static SIF of the CT specimen with crack length of $5 \mathrm{~mm}$. Through the comparison between FEM values and the theoretical values, the FEM model's rationality and validity are proved. Then, based on the previous study, the dynamic finite element is used to calculate the dynamic SIF in the fatigue crack tip under high frequency sinusoidal alternating loading condition. And the relationship between the SIF range and the crack length during the process of fatigue crack growth is found out.

\subsubsection{The Crack Tip Stress Singularity}

The theory of fracture mechanics has illustrated that the stress fields at crack tip possess the singularity. That is, stress components around the crack tip are proportional to $r^{-}$ $1 / 2$, and when $r \rightarrow 0$, the stress increases sharply. In the traditional finite element methods, the internal stress and displacement of element are expressed by matrix polynomial, but it can not properly describe the variation of the stress at the singular point. In general, two methods are presented to solve this problem; one way is to refine the mesh and as a result, more nodes are generated having a great influence on the calculation speed. The other way is to place the singular element near the crack tip, so that it can well reflect the singularity of the stress field. However, the conventional element is still used at the outer side of the singular element. The singularity isoparametric element method is adopted in this paper, that is, by moving the midside-node of the 8-node isoparametric element from the normal position to $1 / 4$ of side length, the stress singularity occurs near the angular point, as shown in section (2) in Fig. (6). In order to well reflect the stress field, four singularity isoparametric elements are placed at crack tip, as shown in Fig. (6).

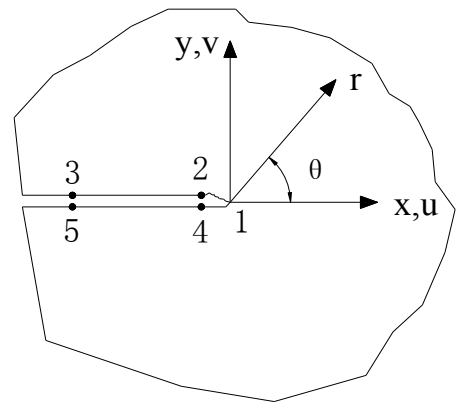

Fig. (5). The local coordinate system of type I crack tip.

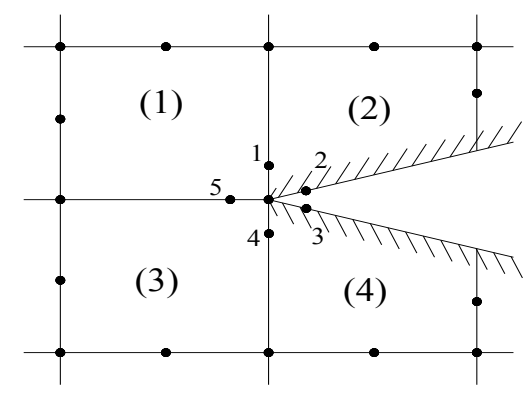

Fig. (6). Four singularity isoparametric elements at crack tip.

\subsubsection{FEM Calculation of Static SIF}

At first, the standard CT specimen with crack length of $5 \mathrm{~mm}$ as shown in Fig. (2) is modeled. The CT specimen is made of $45 \#$ steel with an elasticity modulus of $206 \mathrm{GPa}$, a poisson ratio of 0.27 , and density of $7800 \mathrm{~kg} / \mathrm{m}^{3}$. The detailed steps to create three-dimensional model mesh of CT specimen are described in this section. First, for creating nodes, elements and structure, and for establishing the twodimensional model, a quadrilateral isoperimetric element with 8 nodes of PLANE82 is used, which is degenerated into a triangular isoperimetric element with 6 nodes near the crack tip by ANSYS command KSCON, and the SOLID95 element is used to create the three-dimensional model. The three-dimensional model mesh is presented in Fig. (7). The upper semi-cylinder of the upper loading hole is set to a fixed state. The lower semi-cylinder of the lower loading hole has a stress surface and the uniform distributed pressure of $44.8 \mathrm{MPa}$ is applied to the surface. Before extracting the SIF from the calculation of ANSYS, defining a path by selecting nodes from 1 to 3 on the crack model is necessary, having node 1 at the crack tip, as shown in Fig. (5). Through ANSYS analysis, the static SIF of the CT specimen is 434.67 $N \cdot m^{-3 / 2}$. The theoretical calculation formula of the compact tension specimen is expressed [16] as: 
$K_{I}=\frac{F}{B W^{1 / 2}} g\left(\frac{a}{W}\right)$

$g\left(\frac{a}{W}\right)=\frac{\left(2+\frac{a}{W}\right)\left(0.886+4.64 \frac{a}{W}-13.32\left(\frac{a}{W}\right)^{2}+14.72\left(\frac{a}{W}\right)^{3}-5.6\left(\frac{a}{W}\right)^{4}\right)}{\left(1-\frac{a}{W}\right) 3 / 2}$

Where $F$ is the applied force on the specimen, $B$ is the thickness of the specimen, $W$ is the width of the specimen, $a$ is the length of the crack. According to this formula, the theoretical solution of the SIF of the CT specimen is 445.87 $N \cdot \mathrm{mm}^{-3 / 2}$. The error of static SIF between the calculated result by finite element method and the theoretical result is $2.51 \%$. This proves that the calculation model of CT specimen is correct, mesh well and the results of calculation have a good precision.

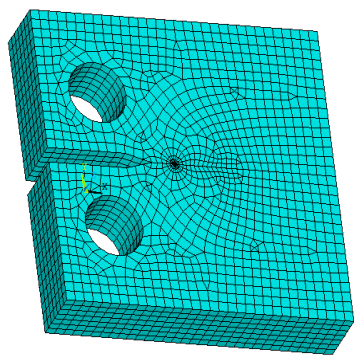

Fig. (7). The three-dimensional model mesh.

\subsubsection{The Variation Law of Dynamic SIF at Crack Tip Under High Frequency Resonant Loading}

The method of establishing model and mesh of the CT specimen is the same as the static case. But, the CT specimen is under high frequency resonant loading with maximum load as $8.35 \mathrm{kN}$, the minimum load as $5.65 \mathrm{kN}$, the average load as $7 \mathrm{kN}$ and the frequency as $125 \mathrm{~Hz}$. Firstly, the static analysis on the CT specimen model under the static loading of $7 \mathrm{kN}$ was performed. The displacement of every node in the direction of crack propagation was extracted. Secondly, the dynamic analysis on the CT specimen model under the sinusoidal loading condition was performed and the displacement of every node in the direction of crack propagation was extracted. Finally, the displacement of the combination results of dynamical and static calculations in every node along the direction of crack propagation can be obtained at any time in a stress cycle. And then putting the values of the displacement into Eq.3, the $K_{I}(t)$ and $r$ can be fitted by least-square principle, and the dynamic SIF value of $r=0$ is obtained by $K_{I}(t)-r$ curve. Thus, the dynamic SIF at crack tip is determined.

The dynamic SIF values at crack tip in one stress cycle during the fatigue crack growth process are shown in Fig. (8). The relation of dynamic SIF amplitudes with the crack length is shown in Fig. (9).

Fig. (8) represents that SIF values at type I fatigue crack tip have the same variation law as applied to load in a stress cycle, which are all sinusoidal changing plots. Fig. (9) represents that SIF amplitudes at type I fatigue crack tip increase with the crack propagation.

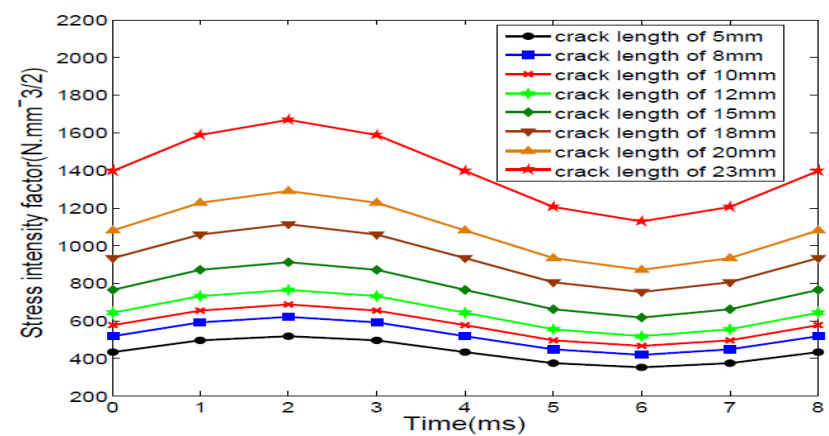

Fig. (8). The dynamic SIFs at crack tip in one stress cycle.

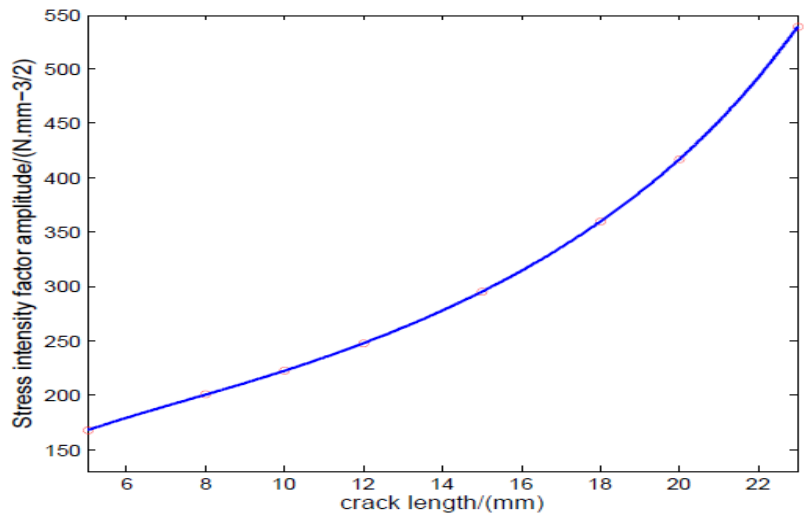

Fig. (9). The relations of SIF amplitudes with the crack length.

\section{THE DISPLACEMENT, STRAIN FIELDS AT CRACK TIP UNDER HIGH FREQUENCY RESO- NANT LOADING}

\subsection{The Variation Law of the Displacement Fields of CT Specimen in one Loading Cycle}

In this section, the variation law of the displacement and strain field at crack tip of CT specimen under high frequency resonant loading in one stress cycle is studied. Firstly, the CT specimen with crack length of $5 \mathrm{~mm}$ is taken as an example, and in this case, the variation laws of the displacement and strain field at crack tip are obtained. Secondly, calculations are carried out on different lengths of the CT specimens. Meanwhile, the result of calculation is also verified. The specimen size and calculating region at crack tip are shown in Fig. (2). The dynamic FEM is performed on the CT specimen, and for the application of load, finite element modeling and the solving method are the same as the calculation of the dynamic SIF, as described in section 2. To directly observe the change of displacement and strain field at crack tip, a MATLAB procedure is applied post-processing. The three-dimension graph of the displacement within the rectangular region at crack tip in a stress cycle is acquired, as shown in Fig. (10). Based on these results, the variation laws of the displacement field of CT specimen in one loading cycle are obtained. It can be observed that all the maximum displacements are located near the point $\mathrm{D}$ of rectangular region, as shown in Fig. (2). All the minimum displacements are located near the point $F$ of rectangular region. This phenomenon could be due to the point $\mathrm{D}$ near the load position 


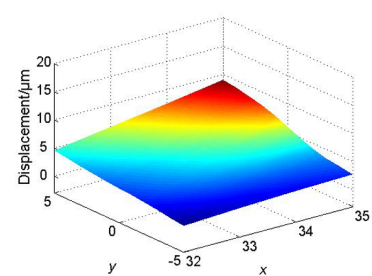

(a) $0 \mathrm{~ms}$

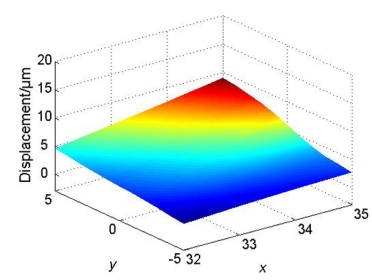

(e) $4 \mathrm{~ms}$

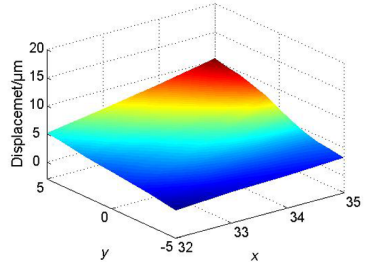

(b) $1 \mathrm{~ms}$

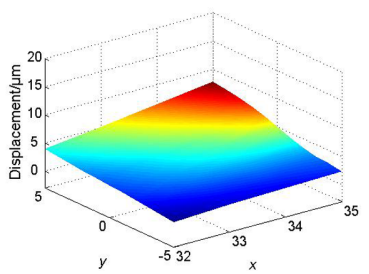

(f) $5 \mathrm{~ms}$
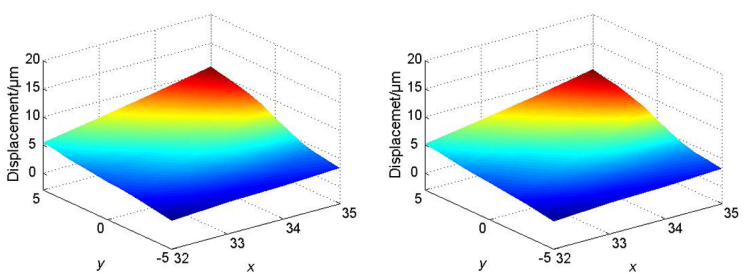

(c) $2 \mathrm{~ms}$

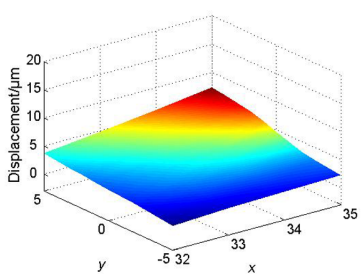

(g) $6 \mathrm{~ms}$ (d) $3 \mathrm{~ms}$

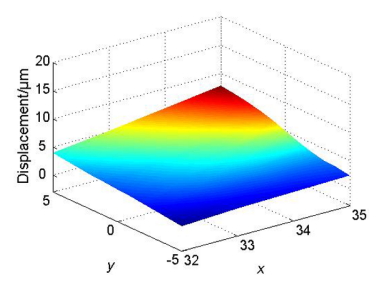

(h) $7 \mathrm{~ms}$

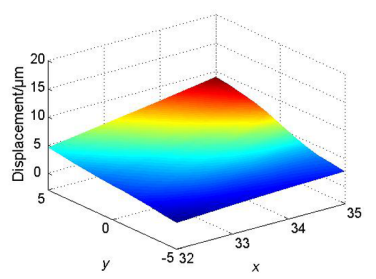

(i) $8 \mathrm{~ms}$

Fig. (10). The crack tip region displacement fields in one load cycle.

and the point $F$ which is far from the load position. Fig. (11) represents that the change of the maximum displacement located at rectangular region point $\mathrm{D}$, the minimum displacement located at rectangular region point $\mathrm{F}$ and the displacement at crack tip in one sinusoidal loading cycle are all sinusoidal, having the same variation trend with the applied load. Calculations indicate that the other points within the rectangular region have the same variation trend with the crack tip.

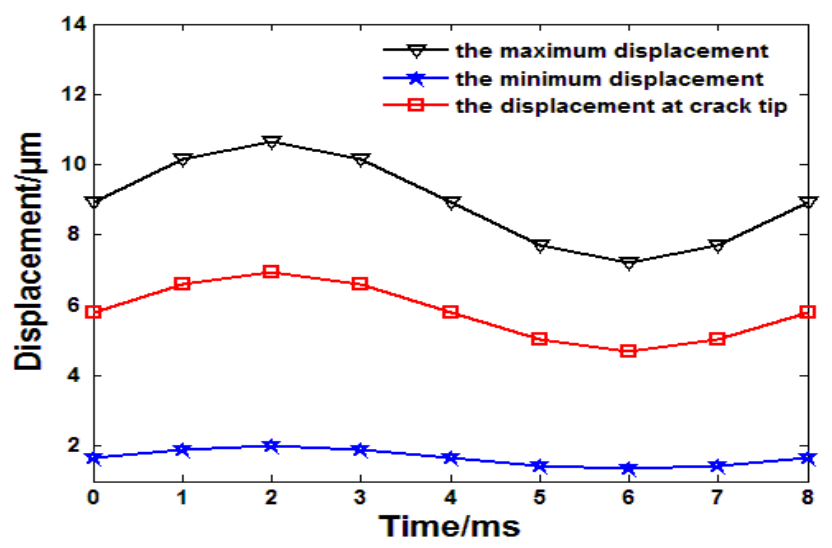

Fig. (11). Maximum, minimum and crack tip displacements in one load cycle.

\subsection{The Variation Law of the Strain Fields of CT Speci- men in One Loading Cycle}

In the same way of the calculation of the displacement field, the variation law of the strain field and the strain at crack tip in a stress cycle are obtained. Fig. (12) represents the change of the strain fields at crack tip in a stress cycle. It can be observed that all the maximum strains are at crack tip; the high strain values field is located near the crack tip, that is, because the crack tip is the place with stress concentration. All the strain fields have the similar shape, however the strain amplitude field shows the strain symmetrical distribution along the direction of crack propagation, and along the direction of crack propagation, the strain value is reduced gradually. Fig. (13) represents that the change of the strain at crack tip over time in a stress cycle is also sinusoidal, having similar variation trend with the applied load. Calculations indicate that the other points within the rectangular region have the same similar variation trend with the crack tip.

\subsection{The Variation Law of the Displacement Amplitude Field During the Process of Fatigue Crack Growth}

The applied load to the CT specimen is sinusoidal in the high frequency resonant fatigue crack propagation test. It is convenient to study the follow variation laws of the displacement field during the process of fatigue crack growth. The displacement amplitude field can be obtained by subtracting the values of the displacement field under maximum load and the values of the displacement field under minimum load in a stress cycle. Fig. (14a-c) indicate that the displacement amplitude field shape is relatively flat at crack tip of CT specimen with crack length of 5,8,10mm. From the rest of the figures, it can be seen that the displacement amplitude fields becoming steeper with the crack propagation. It shows that the change of the displacement amplitude field will be greater with crack propagation. 


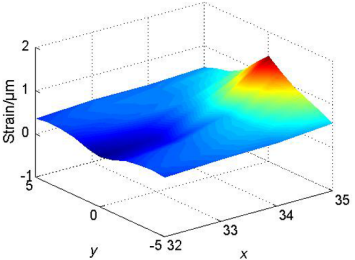

(a) $0 \mathrm{~ms}$

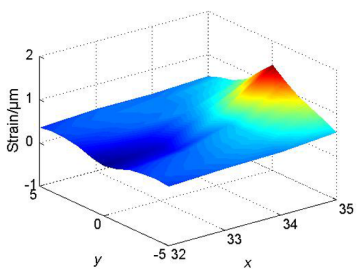

(e) $4 \mathrm{~ms}$

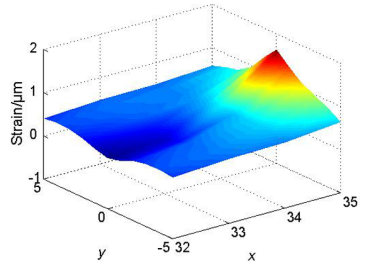

(b) $1 \mathrm{~ms}$

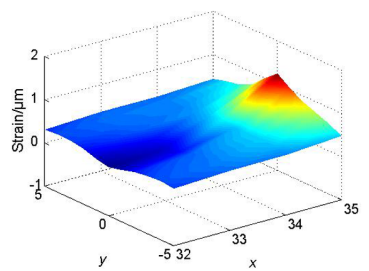

(f) $5 \mathrm{~ms}$

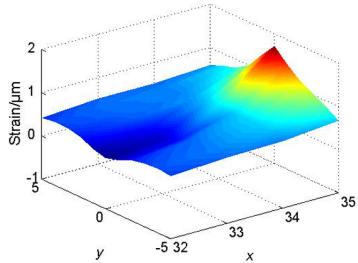

(c) $2 \mathrm{~ms}$

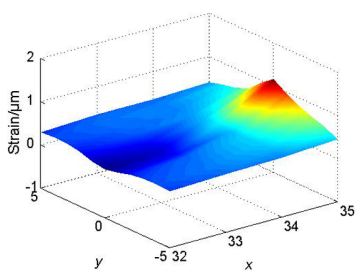

(g) $6 \mathrm{~ms}$

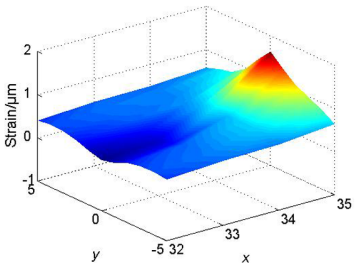

(d) $3 \mathrm{~ms}$

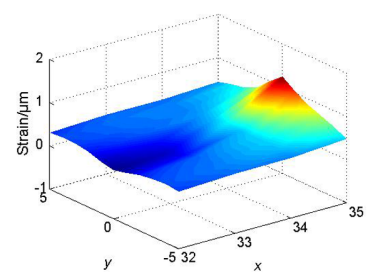

(h) $7 \mathrm{~ms}$

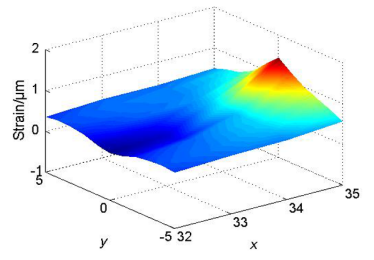

(i) $8 \mathrm{~ms}$

Fig. (12). The strain fields within crack tip region in one load cycle.

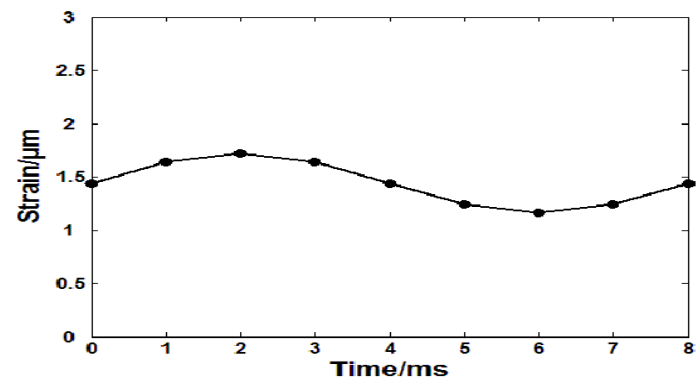

Fig. (13). Crack tip strain in one load cycle.

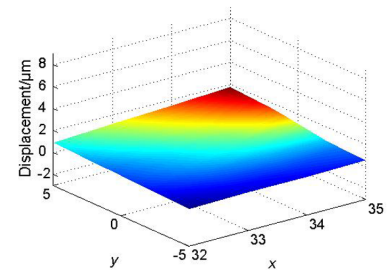

(a) $5 \mathrm{~mm}$

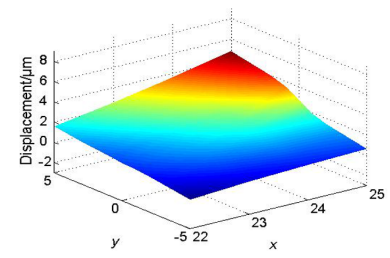

(e) $15 \mathrm{~mm}$

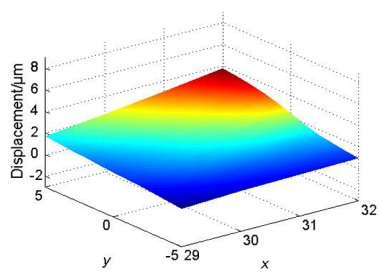

(b) $8 \mathrm{~mm}$

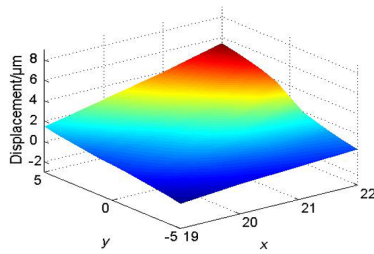

(f) $18 \mathrm{~mm}$

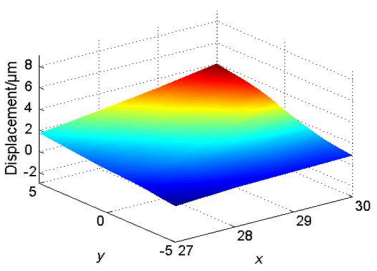

(c) $10 \mathrm{~mm}$

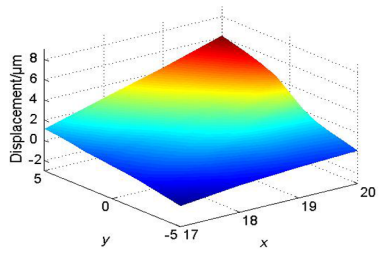

(g) $20 \mathrm{~mm}$

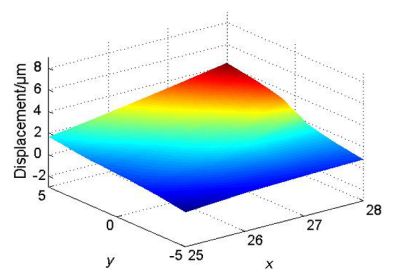

(d) $12 \mathrm{~mm}$

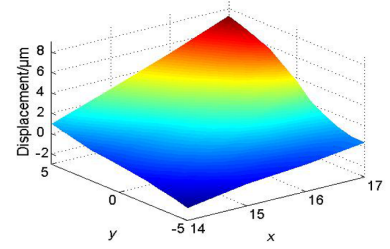

(h) $23 \mathrm{~mm}$

Fig. (14). The displacement amplitude field with different crack length. 


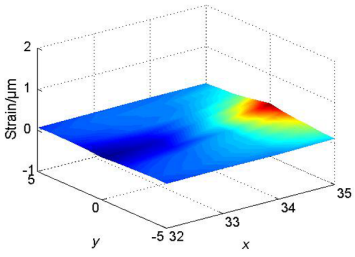

(a) $5 \mathrm{~mm}$

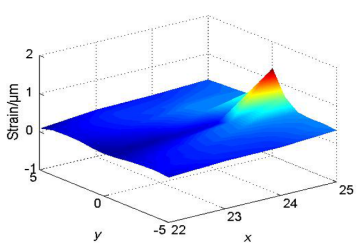

(e) $15 \mathrm{~mm}$

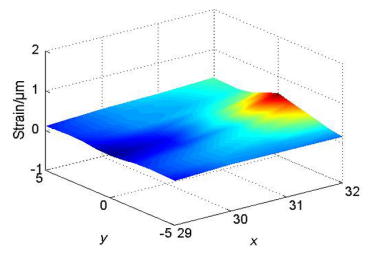

(b) $8 \mathrm{~mm}$

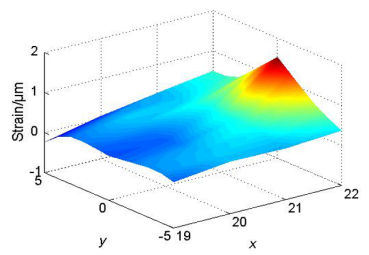

(f) $18 \mathrm{~mm}$

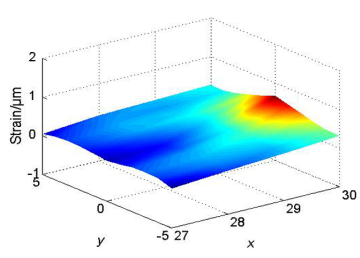

(c) $10 \mathrm{~mm}$

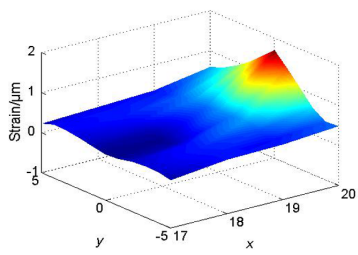

(g) $20 \mathrm{~mm}$

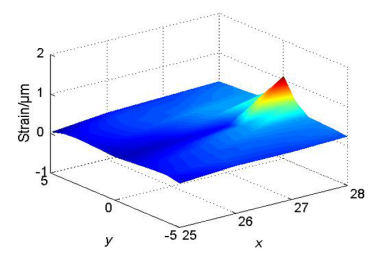

(d) $12 \mathrm{~mm}$

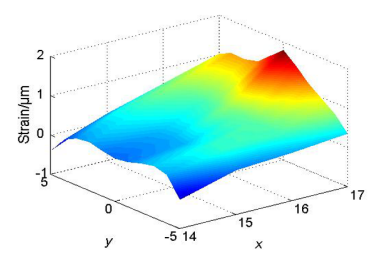

(h) $23 \mathrm{~mm}$

Fig. (15). The strain amplitude field with different crack length.

\subsection{The Variation Law of the Strain Amplitude Field During the Process of Fatigue Crack Growth}

For the convenience of studying the variation law of the strain field during the process of fatigue crack growth, the concept of the strain amplitude field is introduced. The field is obtained by subtracting the values of the strain field under maximum load and the values of the strain field under minimum load in a stress cycle. The strain amplitude fields with different crack lengths are shown in Fig. (15). All the strain amplitude fields have a similar shape; the strain amplitude field shows the strain amplitude symmetrical distribution along the direction of crack propagation,

The high strain amplitude field is located near the crack tip. The strain amplitude is gradually reduced along the direction of crack propagation. Fig. (15a-c) indicate that the strain amplitude field shape is relatively flat at crack tip of CT specimen with crack length of $5,8,10 \mathrm{~mm}$. From the rest of the figures, it can be seen that the strain amplitude fields shape become sharper with the crack propagation, which indicates that the high strain amplitude field increases, and the strain values in the high strain amplitude field are also increase. The fatigue crack can grow easily with the crack propagation.

\section{THE EXPERIMENTAL VERIFICATION OF THE STRAIN VALUE AT CRACK TIP}

In order to verify the accuracy of dynamic finite element method which is used to calculate the strain values at crack tip during crack stable propagation, the fatigue crack propagation tests were performed and the strain values at crack tip point of the CT specimen with crack length of $5 \mathrm{~mm}$ were simultaneously determined using strain gauge. Experiments were carried out on the self-designed electromagnetic resonant fatigue test platform as shown in Fig. (16), including the test loading system, the online crack length measurement system, nature of frequency tracking and test load control system. The test loading system which is used to apply the sine wave dynamic force to the CT specimen, consists of high frequency fatigue tester, specimen, electromagnetic vibrator and amplifying circuit. The crack length online measurement system which is used to measure the fatigue crack length during the process of fatigue crack propagation, consists of CCD camera, optics lens, light source, image acquisition card and computer. The nature of frequency tracking and testing load control system is used to track the systems' nature frequency and control the testing load. The electromagnetic resonant fatigue tester-Hongshan PLG-100 is shown in Fig. (1). Image acquisition card is the PCI-1014, which is produced by NI company. Optics lens is the SONY prime lens of $35 \mathrm{~mm}$. CCD camera is the XC-XT50CE, its image resolution being $734 \mathrm{X} 568$. The specimen material is the $45 \#$ steel after the annealing treatment.

Installing the CT specimen with type I pre-notch on the fatigue tester, the fatigue test is initiated. In this case, the system resonant frequency is $126.4 \mathrm{~Hz}$, the test loadings are set as follows: $F_{\text {max }}=11.65 \mathrm{kN}, F_{\text {min }}=6.35 \mathrm{kN}, F_{m}=9 \mathrm{kN}$, frequency is $126.4 \mathrm{~Hz}$. During the fatigue crack propagation process, the crack size online measurement system is used to measure the fatigue crack length in real-time; nature frequency tracking system and the testing load control system are used to track the resonant frequency and control the testing load. When the crack length reaches $5 \mathrm{~mm}$, the test is stopped. Taking off the specimen, gluing one strain gauge on the surface of CT specimen, the strain gauge position introduced in the paper [17] is shown in Fig. (17), $\theta=54.27^{\circ}$, $r=12.5 \mathrm{~mm}, \phi=68.01^{\circ}$. The dynamic resistance strain gaugeXL2102A is used to measure the dynamic strain at crack tip over time, and its operating frequency is DC $\sim 100 \mathrm{KHz}$. The working frequency of electromagnetic resonant fatigue tester is from $80 \mathrm{~Hz}$ to $300 \mathrm{~Hz}$, so the XL2102 completely meets the criteria. The CT specimen with the $5 \mathrm{~mm}$ fatigue crack length and the resistance gauge is installed on the fatigue tester. In this case, the system resonant frequency is $125 \mathrm{~Hz}$. In order to prevent the rapid crack propagation, the testing load is moved down to $F_{\text {max }}=8.35 \mathrm{kN}, F_{\text {min }}=5.65 \mathrm{kN}, F_{m}=7$ $\mathrm{kN}$, with the rest of the test loading parameters used in the dynamic finite element analysis being the same. The strain values at crack tip point in one stress cycle are obtained by dynamic resistance gauge. The results as shown in Table $\mathbf{1}$ can be obtained by comparing the dynamic FEM calculation and the experimental data. 
Table 1. The results of dynamic FEM calculation and the experimental data in a stress cycle

\begin{tabular}{|c|c|c|c|c|c|c|c|c|c|}
\hline Time (ms) & $\mathbf{0}$ & $\mathbf{1}$ & $\mathbf{2}$ & $\mathbf{3}$ & $\mathbf{4}$ & $\mathbf{5}$ & $\mathbf{6}$ & $\mathbf{7}$ & $\mathbf{8}$ \\
\hline \hline FEM $(\mathrm{mm})$ & 0.001440 & 0.001643 & 0.001712 & 0.001643 & 0.001440 & 0.001246 & 0.001167 & 0.001246 & 0.001440 \\
\hline Test data (mm) & 0.001407 & 0.001599 & 0.001674 & 0.001607 & 0.001406 & 0.001215 & 0.001135 & 0.001221 & 0.001399 \\
\hline Errors (\%) & 2.35 & 2.75 & 2.27 & 2.24 & 2.41 & 2.55 & 2.82 & 2.05 & 2.93 \\
\hline
\end{tabular}

Table 1 indicates that using dynamic FEM to calculate the strain at crack tip has a high precision. By comparing the results of dynamic FEM with the by calculation values of dynamic FEM, experimental data, with the maximum error $2.93 \%$ being obtained, it can be observed that they are higher than the experimental data. This phenomenon could be due to the fact that the strain gradient at crack tip is greater, but only the average strain value from measurement range can be measured by means of strain gauges.

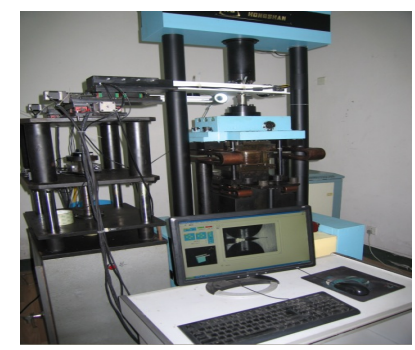

Fig. (16). The image of the experimental facility.

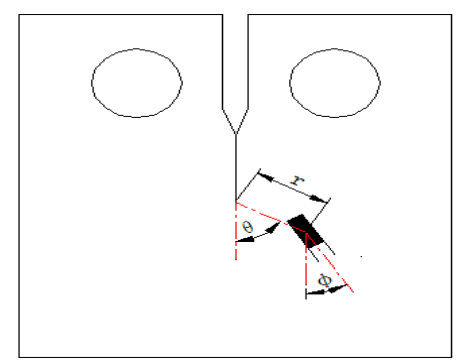

Fig. (17). Arrangement of one strain gauge.

\section{CONCLUSION}

In this study, the static finite element method and the theoretical formula were used to verify the FEM model's validity and the correctness of results. The variation law of displacement, the strain fields and the stress intensity factors at crack tip during the process of fatigue crack propagation test were studied by the dynamic finite element method. Dynamic strain gauges were also used to verify the accuracy of dynamic finite element method applied to calculate the strain at crack tip. Through the analysis and comparison, the following major conclusions can be drawn.

The static finite element method can reliably be used to calculate the static SIF at fatigue crack tip as it has high precision.

The displacement, the strain and SIF at type I fatigue crack tip under the high frequency resonant loading condition are in the same form as the applied load.
The displacement, the strain and SIF amplitude at type I fatigue crack tip under the constant amplitude high frequency resonant loading condition in the stable propagation stage increase with the crack growth.

The dynamic FEM is used to calculate the strain at crack tip as it has high precision.

The research results presented in this paper provide the theoretical base for further investigation of the fatigue crack propagation mechanism and the measurement of crack propagation parameters.

\section{CONFLICT OF INTEREST}

The authors confirm that this article content has no conflict of interest.

\section{ACKNOWLEDGEMENTS}

This work is supported by grants from National Natural Science foundation of China, Grant No.51105338.

\section{REFERENCES}

[1] M. D. Sangid, "The physics of fatigue crack initiation," International Journal of Fatigue, vol. 57, pp. 58-72, 2013.

[2] J. k. Sahu, U. Krupp, and H. J. Christ, '"Fatigue crack initiation behavior in embrittled austenitic-ferritic stainless steel," International Journal of Fatigue, vol. 45, pp. 8-14, 2012.

[3] C. Navarro, J. Vázquez, and J. Domínguez. "3D vs. 2D fatigue crack initiation and propagation in notched plates," International Journal of Fatigue, vol. 58, pp. 40-46, 2014.

[4] M. Giglio, and A. Manes. "Crack propagation on helicopter panel: experimental test and analysis," Engineering Fracture Mechanics, vol. 75, pp. 866-879, 2008

[5] H. Qi, W. Wen, and L. Xun, "Fatigue life prediction and experiment research for composite laminates with circular hole," Journal of Central South University of Technology (English Edition), vol. 11 , no. 1, pp. 19-22, 2005.

[6] J. Lebahn, H. Heyer, and M. Sander, "Numerical stress intensity factor calculation in flawed round bars validated by crack propagation tests," Engineering Fracture Mechanics, vol. 108, pp. 37-49, 2013.

[7] B. K. Lim, L. K. Koay, and G.B. Horizon, "Design optimization and fatigue testing of an electronically-driven mechanicallyresonant cantilever spring mechanism," Materials and Design, vol. 31, no. 8, pp. 4023-4028, 2010.

[8] A. Walcker, D. Weygand, and O. Kraft, "Inertial effects on dislocation damping under cyclic loading with ultra-high frequencies," Materials Science and Engineering, vol. 400, pp. 397-400, 2005.

[9] A.V. Amirkhizi, T. Aref, and N.N. Sia, "Stress-wave energy management through material anisotropy," Wave motion, vol. 47, no. 8 , pp. 519-536, 2010.

[10] R. Goel, M. D. Kulkarni, K. S. Pandya, and N.K. Naik, "Stress wave micro-macro attenuation in ceramic plates made of tiles during ballistic impact," International Journal of Mechanical Sciences, vol. 83, pp. 30-37, 2014.

[11] B. Pan, A. Asundi, H. Xie, and J. Gao, "Digital image correlation using iterative least squares and pointwise least squares for dis- 
placement field and strain field measurements," Optics and Lasers in Engineering, vol. 47, no. 7, pp. 865-874, 2009.

[12] Q. B. Zhang, and J. Zhao, "Determination of mechanical properties and full-field strain measurements of rock material under dynamic loads," International Journal of Rock Mechanics and Mining Sciences, vol. 60, pp. 423-439, 2013

[13] C.-C. Ma, K.-M. Hung, "Exact full-field analysis of strain and displacement for circular disks subjected to partially distributed compressions," International Journal of Mechanical Sciences, vol. 50, no. 2, pp. 275-292, 2008.

[14] P. Dai, J. Yang, H. Li, and Z. Li, "The plasticity-corrected stress intensity factor for plane stress mode I and mode II cracks," Engineering Fracture Mechanics, vol. 128, pp. 231-235, 2014.
[15] Z. Zhou, X. Xu, A. Y.T. Leung, and H. Yi, "Stress intensity factors and T-stress for an edge interface crack by sympletic expansion," Engineering Fracture Mechanics, vol. 102, pp. 334-347, 2013.

[16] J. Yang, The Numerical Simulation and Analysis of Stress and Strain Field at Crack Tip, Dalian University of Technology, Dalian, 2012 (in chinese).

[17] D. Chakraborty, K.S.R.K. Murthy, and D. Chakraborty, "A new single strain gage technique for the accurate determination of mode I stress intensity factor in orthotropic composite materials," Engineering Fracture Mechanics, vol. 124, pp. 142-154, 2014.

Received: December 23, 2014

Revised: February 2, 2015

Accepted: February 27, 2015

(C) Hong-Li et al.; Licensee Bentham Open.

This is an open access articles licensed under the terms of the Creative Commons Attribution-Non-Commercial 4.0 International Public License (CC BY-NC 4.0) (https://creativecommons.org/licenses/by-nc/4.0/legalcode), which permits unrestricted, non-commercial use, distribution and reproduction in any medium, provided that the work is properly cited. 